\title{
Medical therapy reduces arrhythmia recurrence early after AF ablation
}

Researchers from the University of Pennsylvania, USA have demonstrated that antiarrhythmic drug (AAD) therapy during the 6-week period after catheter ablation of atrial fibrillation reduces the incidence of recurrent atrial arrhythmias.

The findings of the 5A Study represent an important advance in the approach to the treatment of these patients. "There was no information in the literature on the use of AADs after ablation" explains investigator Edward Gerstenfeld "even within our own group, practice varied from physician to physician."

Patients with paroxysmal atrial fibrillation who underwent pulmonary vein isolation ( $n=110)$ were randomly assigned to receive an $\mathrm{AAD}$, as prescribed by the treating electrophysiologist, or to no AAD. All participants were given an atrioventricular nodal blocking agent. The majority of patients in the AAD group received class $1 \mathrm{C}$ agents and $66 \%$ received the same AAD that they were taking before ablation.

After 6 weeks, recurrent atrial arrhythmias, including those requiring cardioversion or hospitalization, were significantly less common among patients who received an AAD. In addition, there was no indication that AAD therapy was associated with an increased risk of serious adverse effects. In fact, the trial was terminated early owing to the significant benefit of AAD therapy.

The researchers have now turned their attention to addressing whether the reduction in arrhythmic events persists up to 1 year after ablation, although early results indicate that the benefits of AADs are limited to the short-term.

Alexandra King

Original article Roux, J.-F. et al. Antiarrhythmics after ablation of atrial fibrillation (5A Study). Circulation 120 , 1036-1040 (2009) 